\title{
Moving forward with forward genetic screens
}

\author{
Deep in vivo phenotyping in C. elegans \\ can identify subtle alleles. \\ Most human diseases are not driven by \\ single genes, but instead, arise most likely \\ from subtle and complex interactions \\ between multiple genes and other factors. \\ Identifying these genes and interactions is \\ difficult, precisely because they are often \\ hidden from conventional experimental \\ methods and analysis.
}

Forward genetic screens, for example, have been used for decades in model organisms to understand the genetic basis of biology and disease. While several important genes and their functions have been discovered using forward genetics, reliance on outdated analytical methods is potentially hindering progress. In her lab at the Georgia Institute of Technology, Hang Lu and colleagues at Stanford University have developed an analysis method for deep in vivo phenotyping that makes forward genetic screens from $C$. elegans more sensitive and therefore, more capable of discovering subtle alleles (Nat. Commun. 7, 12990; 2016).

Lu's lab has worked for years developing high-throughput experimental methods, such as microfluidic devices, to help with the physical process of gathering phenotypic data from worms, but realized that at the other end of the experiment-the analysis of data-tools have lagged behind. "In the past, the process of forward genetics was bottlenecked by handling of the animals... but, we now realize that there is a second bottleneck: how do we identify a mutant as a mutant? This is where we think some new and interesting questions lie," explains Lu.

While methods abound for targeting and disrupting specific genes in model organisms, the analytical methods used by most labs to phenotype resulting mutants are not significantly different from those used decades ago. As Lu comments, "labs typically do this by eye, which means they
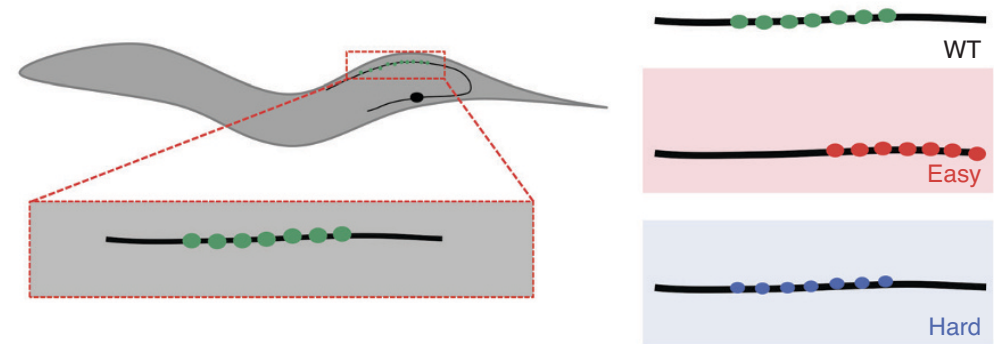

Schematic of synaptic patterning and difficulties in phenotyping subtle mutants. Image adapted from Nat. Commun. 7, 12990; 2016

look at the animals under a microscope and subjectively call out whether an animal is an interesting mutant or not." While this process might work well enough for mutations causing gross disruptions to animal physiology or anatomy, for subtle alleles whose functions are less obvious, mutants might fly under the radar and be missed completely. "At some point, we want to get into problems that are relevant for human diseases, where the phenotype may not be so dramatic anymore," says Lu.

Another important, but often overlooked, component to phenotyping is the role of phenotypic heterogeneity. Even isogenic strains can show considerable variability from animal to animal, and while some groups chalk this up to experimental noise, some of the variance is biological in origin and potentially important. Rather than viewing this "biological noise" as a nuisance, Lu believes that this phenotypic heterogeneity could help shed light on genetic function.

The team's new analysis platform, which relies on the statistical technique of logistic regression, enabled $\mathrm{Lu}$ and her colleagues to address these problems of hidden traits and variability, and identify mutant animals with subtle phenotypic changes, even within heterogeneous populations. Using synaptic patterning in C. elegans as a test case for their analysis, the team imaged GFP-labeled synaptic puncta on the DA9 motor neuron in thousands of wild-type and mutant animals. Rather than just "eyeballing it," for each animal screened, their analysis platform extracted up to 76 features for multidimensional profiling of phenotypic traits.

Wild-type and previously identified strong mutants showed significant levels of variance in single phenotypic featuressuch as puncta size-indicating that previous screens may have missed mutants with more subtle phenotypes. To see how well their logistic regression approach could work at identifying new mutants, the group performed a forward genetic screen and identified several novel alleles affecting synaptic patterning. Consistent with their reasoning for developing a multidimensional analysis platform, they found that subtle mutants could be accurately identified, but only when using multiple features, including population-based statistical features, like differences in variance.

While Lu acknowledges there are limitations for this type of image-based analysis in vivo - adding additional markers for better phenotyping into an animal and imaging pipeline can be challenging-she hopes the group's analytical method will help further increase adoption of C. elegans to help characterize subtle alleles and drug leads that might have more bearing on a variety of human disease states.

Dustin Graham 